\title{
Multi-phase epidemic model by a Markov chain
}

\author{
Stefania Maria Buccellato*, Elisabetta Tornatore \\ Dipartimento di Matematica ed Applicazioni, Università di Palermo, via Archirafi, 34, 90123 Palermo, Italy
}

Received 16 March 2007; received in revised form 23 January 2008

Available online 9 February 2008

\begin{abstract}
In this paper we propose a continuous-time Markov chain to describe the spread of an infective and non-mortal disease into a community numerically limited and subjected to an external infection. We make a numerical simulation that shows tendencies for recurring epidemic outbreaks and for fade-out or extinction of the infection.
\end{abstract}

(c) 2008 Elsevier B.V. All rights reserved.

Keywords: Epidemic models; Markov chain; Numerical simulation

\section{Introduction}

In the last years, a remarkable role in the study of infectious diseases has been played by the use of mathematical models to understand the interplay among various factors that determine the epidemiological process, the disease evolution and the transmission dynamics into a population. The construction of more realistic models has proceeded along two paths, one deterministic and the other stochastic. The deterministic models give information about thresholds, bifurcations and equilibrium phenomenon; the stochastic models play an important role in questions of recurrence and extinction of infections and consider all factors that can profoundly influence the epidemiological state into a population. These models, moreover, provide a method for the characterization of mean probabilistic aspects of diseases such as, for example, the mean value of the infected number.

Recent continuous-time and discrete-time deterministic and stochastic models can be found in Refs. [1,2,8,11-14]. In particular in Ref. [8] the authors consider a deterministic staged-progression model in which infected individuals sequentially pass through a series of stages and derive explicit formulas for reproductive numbers and the endemic steady states.

Statistical data on influenza [3,6], show tendencies for recurring epidemic outbreaks and for fade-out or extinction of the infection (see Figs. 1 and 2). The mechanisms that cause this behaviour, that is one of most important epidemiological aspect, cannot be satisfactorily described by probabilistic indicator as the mean value, the variance or the invariant measure for a stochastic equation. There is a need therefore to search for an appropriate model.

The aim of this paper is to suggest a suitable probabilistic model to show this behaviour and to present a numerical result. The model proposed is a continuous-time Markov chain to describe the spread of an infective and non-mortal disease into a community numerically limited and subjected to an external infection.

\footnotetext{
* Corresponding author. Tel.: +390916040413.

E-mail address: bucci@math.unipa.it (S.M. Buccellato).
} 


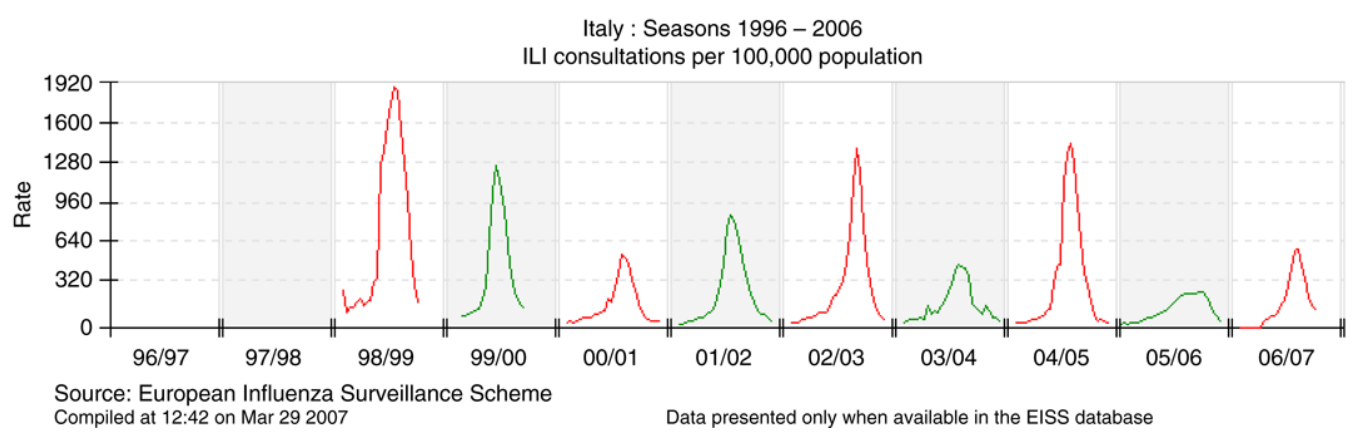

Fig. 1. From European Influenza Surveillance Scheme (EISS): Case reports of influenza in Italy during the season $1996-2006$.

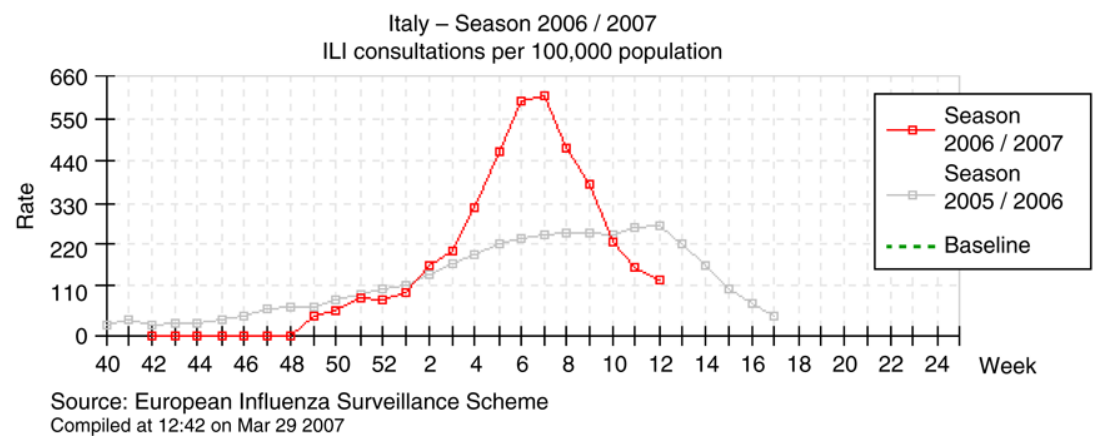

Fig. 2. From European Influenza Surveillance Scheme (EISS): Case reports of influenza in Italy during the season $2006-2007$.

We consider a staged-progression model (like in Ref. [8]) in which the characteristic course of the illness is described by a division of its evolution into several phases that we indicate with $E_{1}, \ldots, E_{m}$ : the first phase can correspond to the incubation period and the last one to the immune period. With $E_{0}$ we indicate the "healthy-susceptible" phase. To simplify we suppose that the path of the illness is uniquely

$$
E_{0} \rightarrow E_{1} \rightarrow E_{2} \rightarrow \cdots \rightarrow E_{m} \rightarrow E_{0}
$$

and we calculate the transition rate among the various phases considering the external contact rate $\varepsilon$, the contact rate $\beta_{j}$ at the phase $E_{j}$ and the average duration $\mu_{j}$ of permanence of an individual at the phase $E_{j}(j=0, \ldots, m)$.

The external contact rate, $\varepsilon$, takes into account that, the transmission of the disease, besides occurring primarily from person to person, both belonging to the target population, can be also be caused by contacts with external reservoirs of infections, with which the susceptible subjects interact when traveling, leading to the phenomenon of "travel-induced" diseases. In this work we suppose that $\varepsilon$ is a positive constant.

The average duration of disease, $\mu_{j}$, and the contact rates, $\beta_{j}$, vary in relation to the disease considered. For example, in the influenza case, adults can spread it from the day before getting symptoms to approximately 5 days after symptoms start; while children can spread influenza for 10 or more days.

The model so obtained reproduces the effect of the individual course of the illness on the collective course. For its generality, moreover, it can be applied in order to analyze several diseases by considering the number of phases and the values of parameters suitable to the illness considered. We present numerical simulations that describe the spread of a disease in a population of size $N$ corresponding to $m$ infection phases. The results confirm the epidemic behaviour characterized by recurring epidemic outbreaks and a long period of fade-out or extinction of the infection. This proves the possibility for developing the program and applying it to interesting epidemiological cases.

\section{Model formulation}

In this section we define the epidemic states of a population and the Markov chain that serves as a model in this paper, by stating the transition rates that the model is based on. Through this paper, $\Omega$ denotes a probability space, $\mathcal{A}$ a $\sigma$-algebra on $\Omega$ and $\mathbb{P}$ a probability measure. 
We consider the diffusion, during a short time period, of an infective disease into a closed population of size $N$ and it is assumed that there are no deaths caused by the disease. We suppose that the path of disease is as in (1).

We denote with $I_{j}(t), j=0,1, \ldots, m$, the number of individuals that are in the phase $E_{j}$ at time $t$. So we have

$$
\sum_{j=0}^{m} I_{j}(t)=N
$$

The epidemic state $\left(I_{0}(t), I_{1}(t), \ldots, I_{m}(t)\right)$ of population at time $t$ can be described by

$$
J(t)=\left(I_{1}(t), \ldots, I_{m}(t)\right),
$$

so, we have

$$
I_{0}(t)=N-\sum_{j=1}^{m} I_{j}(t)
$$

We define the set

$$
Q_{m, N}=\left\{\kappa=\left(k_{1}, \ldots, k_{m}\right) \in\{0,1, \ldots, N\}^{m} \mid \sum_{j=1}^{m} k_{j} \leq N\right\} .
$$

$J(t)$ takes values in $Q_{m, N}$ and the evolution of epidemic state of population is given by the paths of $J(t)$ into the set $Q_{m, N}$.

The cardinality $v_{m}(N)$ of $Q_{m, N}$ is equal to the number of partitions of $N$ indistinguishable individuals into $m+1$ classes and it is equal to

$$
v_{m}(N)=\frac{1}{m !} \prod_{p=1}^{m}(N+p) .
$$

Now, we define the transition rates and for easiness we suppose that the transition among the various phases of disease is regulated by exponential law so that it does not depend on the past.

Moreover we assume that the transition of an individual from a phase $E_{j}(j=1, \ldots, m)$ to the next phase and the transmission of the disease from an infected individual or external sources are independent events.

We let $\mu_{j}$ denote the average duration of an individual at the phase $E_{j}(j=1, \ldots, m)$, so $\frac{1}{\mu_{j}}$ is the parameter of exponential distribution of the duration of an individual at the phase $E_{j}$. More precisely if we denote with $s(t)$ the phase of the disease of an individual at the time $t$, we have

$$
\mathbb{P}\left(\left\{s\left(t^{\prime}\right)=E_{j}, \forall t^{\prime} \in\left[t_{0}, t\left[\mid s\left(t_{0}\right)=E_{j}\right\}\right)=\mathrm{e}^{-\frac{t-t_{0}}{\mu_{j}}}, \quad t \geq t_{0} .\right.\right.
$$

By using (6) the probability that there is a variation of $\delta$ individuals at the phase $E_{j},(j=2, \ldots, m)$, during $[t, t+\Delta t]$ is

$$
\sum_{u-v=\delta}\left(\begin{array}{c}
I_{j-1}(t) \\
u
\end{array}\right)\left(1-\mathrm{e}^{\frac{-\Delta t}{\mu_{j-1}}}\right)^{u}\left(\mathrm{e}^{\frac{-\Delta t}{\mu_{j-1}}}\right)^{I_{j-1}(t)-u}\left(\begin{array}{c}
I_{j}(t) \\
v
\end{array}\right)\left(1-\mathrm{e}^{\frac{-\Delta t}{\mu_{j}}}\right)^{v}\left(\mathrm{e}^{\frac{-\Delta t}{\mu_{j}}}\right)^{I_{j}(t)-v} .
$$

Furthermore, we suppose that at time $t_{0}$ the individual $\sigma_{1}$ be let into the phase $E_{j}$ and the individual $\sigma_{2}$ be let into the phase $E_{0}$, then at time $\tau_{(1 \rightarrow 2)}$ in which $\sigma_{1}$ infects $\sigma_{2}$ we have

$$
\mathbb{P}\left(\left\{\tau_{(1 \rightarrow 2)} \geq t \mid s_{1}\left(t^{\prime}\right)=E_{j}, s_{2}\left(t^{\prime}\right)=E_{0}, \forall t^{\prime} \in\left[t_{0}, \tau_{(1 \rightarrow 2)}[\}\right)=\mathrm{e}^{-\beta_{j}\left(t-t_{0}\right)},\right.\right.
$$

where $s_{i}(t)$ is the phase of disease of the individual $\sigma_{i}(i=1,2)$ at time $t$ and $\beta_{j}$ is the contact rate at the phase $E_{j}$.

We suppose that an individual at the phase $E_{0}$ is infected by external sources at time $\tau$ with probability

$$
1-\mathrm{e}^{-\varepsilon \tau}
$$

where $\varepsilon$ is the external contact rate. 
By using (8) and (9), the probability that there are " $u$ " new individuals at the phase $E_{1}$, during $[t, t+\Delta t]$ is

$$
\left(\begin{array}{c}
I_{0}(t) \\
u
\end{array}\right)\left(1-\mathrm{e}^{-\varepsilon \Delta t} \prod_{j=1}^{m} \mathrm{e}^{-\beta_{j} I_{j}(t) \Delta t}\right)^{u}\left(\mathrm{e}^{-\varepsilon \Delta t} \prod_{j=1}^{m} \mathrm{e}^{-\beta_{j} I_{j}(t) \Delta t}\right)^{I_{0}(t)-u} .
$$

We denote with $\kappa=\left(k_{1}, \ldots, k_{m}\right), \kappa^{\prime}=\left(k_{1}^{\prime}, \ldots, k_{m}^{\prime}\right)$ two elements of $Q_{m, N}$, and we set

$$
\varphi\left(\Delta t ; \kappa, \kappa^{\prime}\right)=\mathbb{P}\left\{J(t+\Delta t)=\kappa^{\prime} \mid J(t)=\kappa\right\}-\delta_{\kappa^{\prime} \kappa}, \quad \kappa, \kappa^{\prime} \in Q_{m, N},
$$

where

$$
\delta_{\kappa^{\prime} \kappa}=1 \text { if } \kappa^{\prime}=\kappa, \quad \delta_{\kappa^{\prime} \kappa}=0 \text { if } \kappa^{\prime} \neq \kappa .
$$

To calculate transition rates we have to compute

$$
\lim _{\Delta t \rightarrow 0} \frac{1}{\Delta t} \varphi\left(\Delta t ; \kappa, \kappa^{\prime}\right)
$$

and we have to examine two cases: $\kappa \neq \kappa^{\prime}$ and $\kappa=\kappa^{\prime}$.

If we take into account the number of individuals changing phase during $[t, t+\Delta t]$, we can introduce the following events:

$$
\begin{aligned}
& B_{0, \Delta t}=\{\text { no individual changes phase during }[t, t+\Delta t]\} \\
& B_{1, \Delta t}=\{\text { only one individual changes phase during }[t, t+\Delta t]\} \\
& B_{2, \Delta t}=\{\text { two or more individuals change phase during }[t, t+\Delta t]\} .
\end{aligned}
$$

Note that $B_{0, \Delta t}, B_{1, \Delta t}, B_{2, \Delta t}$ are two by two disjoint sets and they cover all events

$$
B_{0, \Delta t} \cap B_{1, \Delta t}=B_{0, \Delta t} \cap B_{2, \Delta t}=B_{1, \Delta t} \cap B_{2, \Delta t}=\emptyset \quad B_{0, \Delta t} \cup B_{1, \Delta t} \cup B_{2, \Delta t}=\Omega
$$

and, by virtue of (6)-(10), we have

$$
\lim _{\Delta t \rightarrow 0} \frac{1}{\Delta t} \mathbb{P}\left\{B_{2, \Delta t}\right\}=0 .
$$

Now, if $\kappa \neq \kappa^{\prime}$ we have:

$\left(a_{1}\right) k_{j}^{\prime}=k_{j}-1, k_{j+1}^{\prime}=k_{j+1}+1, k_{l}^{\prime}=k_{l}$ where $l \neq j$ and where $l \neq j+1$;

$\left(a_{2}\right) k_{j}^{\prime}=k_{j}$ where $j=1,2, \ldots, m-1, k_{m}^{\prime}=k_{m}-1$;

$\left(a_{3}\right) k_{1}^{\prime}=k_{1}+1, k_{j}^{\prime}=k_{j}$ where $j=2, \ldots, m$;

$\left(a_{4}\right) \kappa^{\prime} \neq \kappa$ and any previous condition holds.

We observe that

$$
\left\{J(t+\Delta t)=\kappa^{\prime} \mid J(t)=\kappa\right\} \cap B_{0, \Delta t}=\emptyset, \quad \delta_{\kappa^{\prime} \kappa}=0
$$

and by (11) we obtain

$$
\varphi\left(\Delta t ; \kappa, \kappa^{\prime}\right)=\mathbb{P}\left(\left\{J(t+\Delta t)=\kappa^{\prime} \mid J(t)=\kappa\right\} \cap B_{1, \Delta t}\right)+\mathbb{P}\left(\left\{J(t+\Delta t)=\kappa^{\prime} \mid J(t)=\kappa\right\} \cap B_{2, \Delta t}\right) .
$$

In the $\left(a_{1}\right)$ case, by using $(12)$

$$
\varphi\left(\Delta t ; \kappa, \kappa^{\prime}\right)=\mathbb{P}\left(\left\{J(t+\Delta t)=\kappa^{\prime} \mid J(t)=\kappa\right\} \cap B_{1, \Delta t}\right)=k_{j}\left(1-\mathrm{e}^{-\frac{\Delta t}{\mu_{j}}}\right)
$$

and

$$
\lim _{\Delta t \rightarrow 0} \frac{1}{\Delta t} \varphi\left(\Delta t ; \kappa, \kappa^{\prime}\right)=\frac{k_{j}}{\mu_{j}}
$$


In the $\left(a_{2}\right)$ and $\left(a_{3}\right)$ cases we obtain

$$
\lim _{\Delta t \rightarrow 0} \frac{1}{\Delta t} \varphi\left(\Delta t ; \kappa, \kappa^{\prime}\right)=\frac{k_{m}}{\mu_{m}},
$$

and

$$
\lim _{\Delta t \rightarrow 0} \frac{1}{\Delta t} \varphi\left(\Delta t ; \kappa, \kappa^{\prime}\right)=\left(\varepsilon+\sum_{j=1}^{m} \beta_{j} k_{j}\right)\left(N-\sum_{j=1}^{m} k_{j}\right)
$$

respectively.

Finally in the $\left(a_{4}\right)$ case we observe that if $J(t)=\kappa$ and if there is only one individual changing phase during $[t, t+\Delta t]$, the state $\kappa^{\prime}=J(t+\Delta t)$ must satisfy only one of the $\left(a_{1}\right),\left(a_{2}\right),\left(a_{3}\right)$ cases. While, if $\kappa^{\prime} \neq \kappa$ and the $\left(a_{1}\right)$, $\left(a_{2}\right),\left(a_{3}\right)$ cases do not hold we have

$$
\left\{J(t+\Delta t)=\kappa^{\prime} \mid J(t)=\kappa\right\} \cap B_{0, \Delta t}=\left\{J(t+\Delta t)=\kappa^{\prime} \mid J(t)=\kappa\right\} \cap B_{1, \Delta t}=\emptyset
$$

and, by using (11) and (12) we have

$$
\lim _{\Delta t \rightarrow 0} \frac{1}{\Delta t} \varphi\left(\Delta t ; \kappa, \kappa^{\prime}\right)=0 .
$$

Now, if $\kappa=\kappa^{\prime}$, using total probability theorem we deduce that

$$
\lim _{\Delta t \rightarrow 0} \frac{1}{\Delta t} \varphi\left(\Delta t ; \kappa, \kappa^{\prime}\right)=-\left(\varepsilon+\sum_{j=1}^{m} \beta_{j} k_{j}\right)\left(N-\sum_{j=1}^{m} k_{j}\right)-\sum_{j=1}^{m} \frac{k_{j}}{\mu_{j}} .
$$

\section{Markov chain}

By virtue of (13)-(17) we can affirm that the random variable $J(t)$ is a continuous-time Markov chain (about continuous-time Markov chain see Ref. [7]) which takes values in $Q_{m, N}$. The family of transition matrix is given by

$$
\mathrm{e}^{(t-s) B}, \quad 0 \leq s \leq t,
$$

where $B$ is a square matrix $v_{m}(N) \times v_{m}(N)$,

$$
B=\left(b_{\kappa^{\prime} \kappa}\right)_{\kappa^{\prime} \kappa \in Q_{m, N}} .
$$

Elements $b_{\kappa^{\prime} \kappa}$ are so defined that the considerations on transition rates which are calculated in previous section are confirmed. An element

$$
\kappa=\left(k_{1}, k_{2}, \ldots, k_{m}\right) \in Q_{m, N}
$$

is fixed and if $\kappa^{\prime} \in Q_{m, N}$, we set

$$
\begin{aligned}
& b_{\kappa^{\prime} \kappa}=\left(\varepsilon+\sum_{j=1}^{m} \beta_{j} k_{j}\right)\left(N-\sum_{j=1}^{m} k_{j}\right) \quad \text { if } \kappa^{\prime}=\left(k_{1}+1, k_{2}, \ldots, k_{m}\right), \\
& b_{\kappa^{\prime} \kappa}=\frac{k_{j}}{\mu_{j}} \quad \text { if } \kappa^{\prime}=\left(k_{1}, \ldots, k_{j-1}, k_{j}-1, k_{j+1}+1, k_{j+2}, \ldots, k_{m}\right), \\
& b_{\kappa^{\prime} \kappa}=\frac{k_{m}}{\mu_{m}} \text { if } \kappa^{\prime}=\left(k_{1}, \ldots, k_{m-1}, k_{m}-1\right), \\
& b_{\kappa^{\prime} \kappa}=-\left(\varepsilon+\sum_{j=1}^{m} \beta_{j} k_{j}\right)\left(N-\sum_{j=1}^{m} k_{j}\right)-\sum_{j=1}^{m} \frac{k_{j}}{\mu_{j}} \quad \text { if } \kappa^{\prime}=\kappa, \\
& b_{\kappa^{\prime} \kappa}=0 \quad \text { otherwise. }
\end{aligned}
$$


Let $J(0)=J_{0}$ be the random variable in $Q_{m, N}$ which represents the epidemic state of population at time $t=0$. To represent the laws of $J(0)$ and of $J(t)$ by vectors we set

$$
S_{v_{m}(N)}=\left\{v=\left(v_{1}, \ldots, v_{v_{m}(N)}\right) \in \mathbb{R}^{v_{m}(N)} \mid v_{q} \geq 0, q=1, \ldots, v_{m}(N), \sum_{i=1}^{v_{m}(N)} v_{q}=1\right\} .
$$

Let $v(0) \in S_{v_{m}(N)}$ be the vector that represents the law of $J(0)$. The vector $v(t)$ that represents the law of $J(t)$ is defined as

$$
v(t)=\mathrm{e}^{t B} v(0)
$$

and, by using (18)-(22), we obtain that $v(t) \in S_{v_{m}(N)}$. We have

$$
v(t)=\mathrm{e}^{(t-s) B} v(s), \quad 0 \leq s \leq t
$$

and moreover

$$
\lim _{\Delta t \rightarrow 0} \frac{1}{\Delta t}(v(t+\Delta t)-v(t))=\frac{\mathrm{d}}{\mathrm{d} t} v(t)=B v(t) .
$$

For all $v(0)$, when $t$ goes to $+\infty$, the vector $v(t)$ converges to invariant law $\bar{v}=\left(\bar{v}_{1}, \ldots, \bar{v}_{v_{m}(N)}\right) \in S_{v_{m}(N)}$; the uniqueness of the invariant law $\bar{v}$ follows by positivity of coefficients $\varepsilon$ and $\frac{1}{\mu_{j}}$. Moreover we have $\overline{v_{q}}>0$ for $q=1, \ldots, v_{m}(N)$, so, if $\bar{J}$ is a random variable having the law $\bar{v}$, then for all $\kappa \in Q_{m, N}$ we have $\mathbb{P}\{\bar{J}=\kappa\}>0$.

\section{Numerical simulation}

By the numerical point of view, the vector $v(t)$ defined in (23) supply all information about the epidemiological state of the population. To describe the most important aspect, namely the possibility to reproduce the epidemic behaviour characterized by recurring epidemic outbreaks and for long period of fade-out or extinction of the infection, it needs to use numerical programs able to reproduce stochastic situations.

Our numerical simulation has been made using Matlab and shows the possibility to develop this program and to apply it to interesting cases from an epidemiological point of view. By using a time-discretization, the continuous-time Markov chain $J(t)$ must be replaced from discrete-time Markov chain $J_{1}(t)$

$$
t=0, \alpha, 2 \alpha, \ldots, n \alpha, \ldots
$$

If we consider $\alpha$ sufficiently small so that

$$
\mathbb{P}\left(B_{2, \alpha}\right) \ll \mathbb{P}\left(B_{1, \alpha}\right),
$$

the transition matrix of the Markov chain can be approximated by $R=\alpha B+I$ where $B=\left(b_{\kappa^{\prime} \kappa}\right)$ is the matrix defined in (18)-(22). In the numerical simulation we redefine the unit time to be $\alpha=1$.

We assume that there are $m$ epidemic phases. To label the states $\kappa=\left(k_{1}, \ldots, k_{m}\right) \in Q_{m, N}$ with $1,2, \ldots v_{m}(N)$ we use the function

$$
\Lambda\left(\left(k_{1}, \ldots, k_{m}\right)\right)=1+\sum_{p=1}^{m} \frac{1}{p !}\left(\prod_{q=1}^{p}\left(N+q-\sum_{j=1}^{m-p} k_{j}\right)-\prod_{q=1}^{p}\left(N+q-\sum_{j=1}^{m-p+1} k_{j}\right)\right) .
$$

It is easy to verify that $\Lambda(\cdot)$ is a one-to-one correspondence between the state space $Q_{m, N}$ and the set $\left\{1, \ldots, v_{m}(N)\right\}$.

The numerical simulation reproduces the epidemic behaviour and can be applied in order to analyze several diseases within a numerically limited community, naturally by considering the number of phases and the values of the parameters suitable to the illness considered.

Model parameters can be estimated from available demographic and epidemiological data. For example, in the influenza case, the average duration of infection and distribution of infectious period can be estimated from the European Influenza Surveillance Scheme (EISS) [6] or from the Centers for Disease Control (CDC) [3].

We assume that the contact rates $\beta_{i}(i=i, \ldots, m)$ and the external contact rate $\varepsilon$ depend on the structure and size of the population being considered, in particular they are inversely proportional to the population size (see, for 


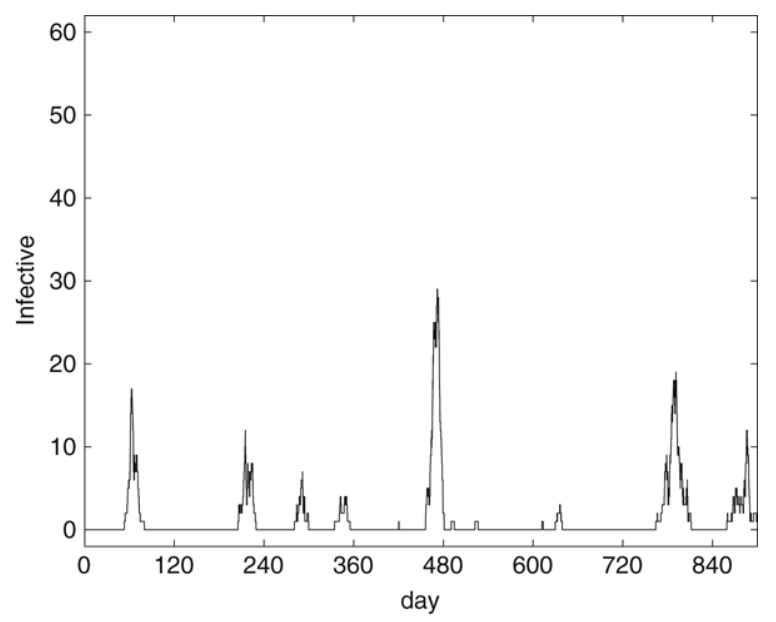

Fig. 3. Evolution in the time of infective individuals within a population of size $N=60$ when $m=3$ and $\beta_{1}=\beta_{2}=5.6 \times 10^{-3}, \beta_{3}=0$, $\varepsilon=3.47 \times 10^{-4}, \mu_{1}=\mu_{2}=2.26$ days, $\mu_{3}=11.31$ days.

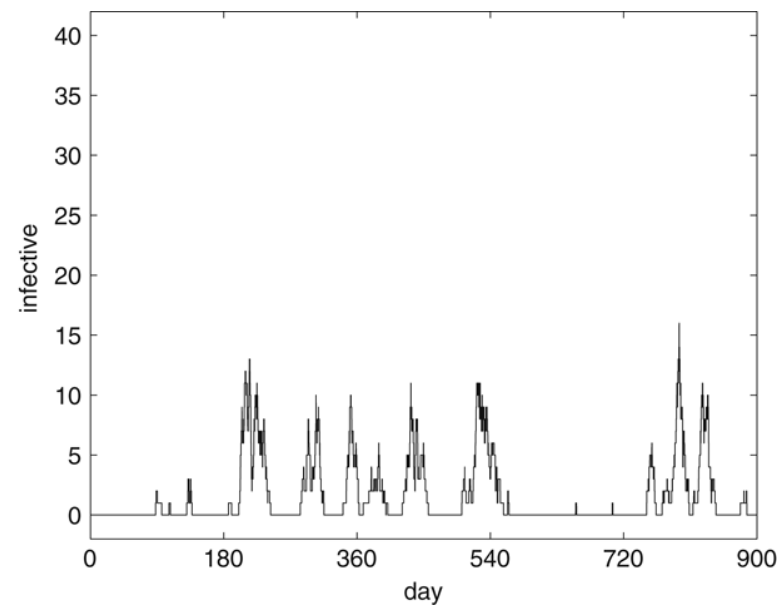

Fig. 4. Evolution in the time of infective individuals within a population of size $N=40$ when $m=4$ and $\beta_{1}=\beta_{2}=\beta_{3}=8.7 \times 10^{-3}, \beta_{4}=0$, $\varepsilon=5.2 \times 10^{-4}, \mu_{1}=\mu_{2}=\mu_{3}=1.49$ days, $\mu_{4}=7.49$ days.

instance, Ref. [5]). This is natural because they depend on the probability that an encounter between susceptible and infective individual or source results in the transmission of the disease and on the rate at which such encounters occur.

The numerical simulation has been made considering the cases of $m=3, m=4$ phases of infection within a population of size $N=60, N=40$, respectively and choosing our parameters so that the total time is $T=2.5$ years and the mean duration of the infection is 14 days. The results in Figs. 3 and 4 show the number of infective individuals in the time. We observe that the qualitative behaviour reproduces what was obtained with experimental data (see Figs. 1 and 2) and reflects, in a way, the expected behaviour.

\section{Conclusions}

Dynamic models of infectious diseases for studying disease transmission and persistence are important to work out the control, prevention or intervention strategies. Many epidemic diseases, like influenza, when introduced into the host population, break out and burn out locally. In this study we present a model to cover these epidemic dynamics. We consider the spread of an infective and non-mortal disease within a closed community numerically limited and subjected to an external infection and we subdivide a single infectious class of a SIS model into $m$ phases such that a newly infected individual enters the first phase, passes through each successive phase and then goes back to the 
susceptible class. The qualitative behaviour obtained from numerical simulations agrees with that from experimental data and shows extinction and recurrence of epidemic.

In our paper we assumed that the external contact rate $\varepsilon$ is constant; in literature, it is related to "travel-induced" diseases, in fact travel facilitates the physical transport of pathogens and vectors and increases the exposure of individuals to disease. It would be interesting to examine the parameter $\varepsilon$, modeled as an increasing function of the infectious number, influence the behaviour of the model in a population.

The recurrence of fluctuations and the non-extinction of the disease are related to the noise present in biological systems, in Ref. [4] the authors introduce a pulse source with memory to describe the number of contacts between ill and healthy people, where the delay describes the incubation period of the illness. So they compare development of illness with different incubation periods.

The results obtained in this work are strongly dependent on the choice of the exponential law; this mathematical assumption is used by numerous authors. Interesting works exist about the distribution of infectious periods in disease models, such as measles, which pay particular attention to the difference between exponentially distributed and constant periods $[9,10]$.

\section{Acknowledgments}

The authors wish to thank Prof. Hisao Fujita Yashima of the University of Torino for his suggestions that have been essential for the realization of this work and also the referees for their useful comments.

\section{References}

[1] L. Allen, A.M. Burgin, Comparison of deterministic and stochastic SIS and SIR models in discrete time, Mathematical Biosciences 163 (2000) 1-33.

[2] R.M. Anderson, R.M. May, Infectious Disease of Humans, Oxford University Press, 1991.

[3] CDC (Centers for Disease Control), The Influenza (Flu) Viruses. http://www.cdc.gov/flu/weekly/weeklyarchives2005-2006/0506summary.htm.

[4] O. Chichigina, D. Valenti, B. Spagnolo, A simple noise model with memory for biological systems, Fluctuation and Noise Letters 5 (2) (2005) 243-250.

[5] M.C.M. De Jong, O. Diekmann, H. Heesterbeek, How does transmission of infection depend on population size? in: Epidemic Models: Their Structure and Relation to Data, vol. 5 (2), Cambridge University Press, Cambridge, UK, 1995, pp. 84-94.

[6] EISS (European Influenza Surveillance Scheme). http://www.eiss.org/index.cgi.

[7] I.I. Guikhman, A.V. Skorokhod, Introduction à la thèorie des processus alèatoires, (traduit du russe), Mir, Moscou, 1980.

[8] J.M. Hyman, J. Li, E.A. Stanley, The differential infectivity and staged progression models for transmission of HIV, Mathematical Biosciences 155 (1999) 77-109.

[9] M.J. Keeling, B.T. Grenfell, Effect of variability in infection period on the persistence and spatial spread of infectious diseases, Mathematical Biosciences 147 (1998) 207-226.

[10] A.L. Lloyd, Realistic distributions of infectious periods in epidemics models: Changing patterns of persistence and dynamics, Theoretical Population Biology 60 (2001) 59-71.

[11] R.K. McCormack, L.J.S. Allen, Disease emergence in deterministic and stochastic models for host and pathogens, Applied Mathematics and Computation 168 (2005) 1281-1305.

[12] I. Nassel, Stochastic models of some endemic infections, Mathematical Biosciences 179 (2002) 1-19.

[13] M.K. Oli, M. Venkataraman, P.A. Klein, L.D. Wendland, M.B. Brown, Population dynamics of infectious diseases: A discrete time model, Ecological Modelling 198 (1-2) (2006) 183-194.

[14] E. Tornatore, S.M. Buccellato, P. Vetro, Stability of a stochastic SIR system, Physica A 354 (2005) 111-126. 